\title{
Intraoperative Urine Output Is Associated with Postoperative Outcome in Pediatric Population Undergone Major Abdominal Operations
}

This article was published in the following Dove Press journal:

Therapeutics and Clinical Risk Management

\author{
Chao Zheng ${ }^{1,2}$ \\ Chunbao Guo iD ${ }^{2,3}$ \\ 'Department of Orthopedics, Children's \\ Hospital, Chongqing Medical University, \\ Chongqing 4000 I4, People's Republic of \\ China; ${ }^{2}$ Ministry of Education Key \\ Laboratory of Child Development and \\ Disorders, National Clinical Research \\ Center for Child Health and Disorders, \\ China International Science and \\ Technology Cooperation Base of Child \\ Development and Critical Disorders, \\ Chongqing Key Laboratory of Pediatrics, \\ Chongqing Engineering Research Center \\ of Stem Cell Therapy, Children's Hospital \\ of Chongqing Medical University, \\ Chongqing, People's Republic of China; \\ ${ }^{3}$ Department of Pediatric General \\ Surgery and Liver Transplantation, \\ Children's Hospital, Chongqing Medical \\ University, Chongqing, People's Republic \\ of China
}

Correspondence: Chunbao Guo Department of Pediatric General Surgery and Liver Transplantation, Children's Hospital, Chongqing Medical University, I36 Zhongshan 2nd Rd, Chongqing 4000 I4, People's Republic of China Tel +86-23-6363l839

Fax +86-23-63622874

Email guochunbao@foxmail.com
Aim: Few data support the advantage of confirming a low urine output target during Rouxen-Y hepaticojejunostomy, which was widely used as an indication for fluid administration. We aimed at evaluating postoperative outcomes in terms of urine output in pediatric patients undergoing elective Roux-en-Y hepaticojejunostomy.

Methods: We retrospectively reviewed 689 patients who had undergone Roux-en-Y hepaticojejunostomy between January 2007 and August 2014 at the Children's Hospital of the Chongqing Medical University. Patients were dichotomized according to the average amount of corrected urine output $\left(6.01 \mathrm{~mL} / \mathrm{kg}^{*} \mathrm{~h}\right)$ as a cut-off point. The primary endpoint was the occurrence of renal complications. The secondary endpoints included prompt postoperative gastrointestinal function recovery, postoperative complications and hospital length of stay.

Results: The lower urine output had a proportional association with lesser amounts of crystalloid fluids $\left(12.99 \pm 6.52\right.$ and $17.36 \pm 7.74 \mathrm{~mL} / \mathrm{kg}^{*} \mathrm{~h}$ for low and high urine output, respectively, $\mathrm{p}=0.006$ ). For patients with a lower urine output, there were trends toward lower incidence rates of grade II postoperative complications (OR, 0.68; 95CI, 0.45-1.03; $\mathrm{p}=0.041)$ and accelerated recovery of gastrointestinal function, as indicated by the first flatus $(\mathrm{p}=0.015)$ and first bowel movement $(\mathrm{p}=0.008)$; however, the occurrence of renal complications did not show significant differences between the groups. The total length of hospital stay was shorter in patients with low urine output ( $7.59 \pm 1.24$ days) than that in patients with a high urine output $(8.01 \pm 2.31$ days, $\mathrm{p}=0.016)$.

Conclusion: Lower urine output is associated with a lower incidence rate II postoperative complications and accelerated recovery of gastrointestinal function, without increasing the occurrence of renal complications in pediatric patient undergone hepaticojejunostomy. The optimal amount of urine output and associated fluid administration should be further investigated.

Keywords: intraoperative urine output, Roux-en-Y hepaticojejunostomy, postoperative recovery, acute kidney injury

\section{Introduction}

During or after surgical procedures, fluid administration might affect postoperative organ function and outcomes. ${ }^{1-3}$ Excessive fluid administration may be responsible for a decrease in muscular oxygen tension and delayed recovery of gastrointestinal function and may be associated with postoperative complications, organ failure or poor survival. On the other hand, restrictive fluid resuscitation may increase the incidence of hypovolemia and hence hypoperfusion, thereby increasing the risk of acute kidney injury (AKI) or other postoperative complications. ${ }^{4,5}$ It is therefore 
recommended to optimize the volume of infused fluid, especially crystalloids, to maintain an appropriate cardiac output and improve outcomes after major surgery. However, the optimal amount of fluid to be administered is unclear.

In the current clinical routine, heart rate (HR), mean arterial pressure (MAP) and central venous pressure (CVP) have been used to assess and guide hemodynamic management practices for patients undergoing major surgery. However, because indwelling urinary catheters allow accurate hourly measurement of urine output during the perioperative period, urinary output has been most widely used as an indicator of intravascular volume status in Europe and in the United States. ${ }^{6,7}$ Usually, to avoid renal hypoperfusion and impending $\mathrm{AKI}$, it is a standard practice to achieve the minimum urine output threshold of $0.5 \mathrm{~mL} / \mathrm{kg} / \mathrm{h} .^{8}$ Recent studies have suggested that a lower urine output of $0.2 \mathrm{~mL} / \mathrm{kg} / \mathrm{h}$ may be physiological and an integral part of the surgical stress response. ${ }^{9}$ Renal reabsorption of water and electrolytes increases in critical conditions, leading to a sharp decrease in urine output, ${ }^{10}$ to only $15 \%$ to $20 \%$ of that found in normal conditions, as modulated through antidiuretic hormone and other mechanisms. In a recent study, implementation of a low urine output guide intraoperative fluid therapy has resulted in improved outcomes, ${ }^{9,11}$ suggesting that the increased fluid volumes required to increase urine output to the standard perioperative target may be harmful.

To better evaluate the role of intraoperative urine output in pediatric surgical practices, we assessed the association between postoperative clinical outcomes and urine output for pediatric patients undergoing pediatric elective Roux-en-Y hepaticojejunostomy, with the belief that a lower urine output in the current clinical practice, representing less fluid administration, is associated with better clinical outcomes and a non-inferior risk of renal complications.

\section{Materials and Methods}

We performed a retrospective analysis of the medical records of pediatric patients who underwent elective Roux-en-Y hepaticojejunostomy at Chongqing Children's Hospital (urban tertiary care teaching hospital) between January 2007 and August 2014. The Ethics Committee of the Chongqing Medical University gave expedited approval of this protocol. All patients undergoing the elective Roux-en-Y hepaticojejunostomy upon meeting the following inclusion criteria were enrolled in this study: age $>1$ year and $<10$ years; no severe sepsis (normal C-reactive protein); and no steroid or immunosuppressive medication administration. Exclusion criteria included evidence of metastatic disease; coagulopathy or platelet dysfunction; preoperative renal dysfunction (creatinine $>50 \%$ upper limit of reference range); application of colloidal infusion solutions within the past $24 \mathrm{~h}$ before surgery. To minimize selection bias, we also excluded urgent and emergency procedures. All the procedures were performed by attending surgeons and stored in the database containing information on these procedures.

\section{Anesthesia Management}

The intraoperative management decisions, including fluid administration and blood products transfusion, were not standardized and were dictated by the hemodynamic parameters at the discretion of the attending trauma surgeon. The hemodynamic parameters, including heart rate, invasive blood pressure, CVP, and urine output, were monitored during the surgical procedures. Patients usually received $3 \mathrm{~mL} / \mathrm{kg} / \mathrm{h}$ of Ringer's lactate solution (RL) throughout the intraoperative period. When low blood pressure (according to the age) was encountered, fluid boluses ( $250 \mathrm{~mL} \mathrm{RL}$ ) were further administered. If blood pressure did not improve effectively, pharmacologic norepinephrine support was used.

Ventilation was performed to maintain $\mathrm{SaO}_{2}>95 \%$ and end-tidal $\mathrm{CO}_{2}$ tensions of $35-40 \mathrm{mmHg}$. Arterial and central venous blood samples were taken hourly for acid-base balance analysis, blood count and basic biochemical laboratory tests. Blood products were transfused according to the ASA practice guidelines transfusion protocol. Packed red blood cells (RBC) were transfused when hemoglobin levels fell below $80 \mathrm{~g} / \mathrm{L}$. On postoperative day (PODs) 1 and before discharge, hematocrit, electrolytes, blood urea, and creatinine serum concentrations were also measured. Additional blood tests, electrocardiography, and measurements of cardiac enzymes were performed as clinically indicated. To roughly assess the fluid load received in the operating room (apart from blood loss and transfusions), we computed the fluid balance according to the following formula, fluid balance $=[$ Crystalloid $+1.5 *($ colloid + blood product)] (Volume In)-[Urine Output $+3 *$ (estimated blood loss)] (Volume Out) - [Operative duration $(\mathrm{hrs}) *($ weight $[\mathrm{kg}]+40)]$ (Maintenance). ${ }^{12}$

\section{Data Collection and Definitions}

Electronic medical records were thoroughly reviewed by investigators who had undergone specific training and 
abstracted the relevant data pertaining to the demographic characteristics (age, sex, admission weight, comorbid conditions), biochemical profiles (hemoglobin, blood glucose, creatinine, serum electrolytes, albumin, retinol binding protein (RBP), C-reactive protein (CRP) and coagulation profiles), intraoperative data (American Society of Anesthesiology (ASA) classification, duration of operation, amount of intraoperative crystalloid and colloid fluid, operating time, intraoperative estimated blood loss (EBL), intraoperative blood transfusion, hemoglobin levels, urine output), and postoperative outcomes (nasogastric tube stay, parenteral nutrition duration, complications (any), hospital length of stay (LOS), intensive care unit (ICU) admission, mortality and necessity for reoperation).

The primary endpoint of interest was the occurrence of renal complications [renal dysfunction defined as creatinine $>50 \%$ upper limit of normal values] on PODs 1 through 5 or AKI. AKI was defined according to the AKIN criteria as mentioned previously. ${ }^{13}$ The secondary endpoints included prompt postoperative gastrointestinal function recovery, postoperative complications and hospital length of stay (the days from operation to discharge). Gastrointestinal symptoms were recorded for the first 5 days postoperatively, including the first postoperative flatus or defecation, gastric retention, nausea or vomiting, time to normal diet, abdominal bloating and/or cramps. All surgical complications were recorded and ranked according to the Clavien-Dindo classification system. This study only included grade II complications or higher, such as wound infection, intra-abdominal abscesses, pneumonia, bacteremia, gastrointestinal bleeding, renal failure and respiratory failure.

\section{Statistical Analysis}

The coefficient of variation (COV) was calculated to measure the dispersion of urine output by dividing the standard deviation by its mean. Continuous data are presented as the means \pm (standard deviations [SD]) for normally distributed data and medians (interquartile ranges) for unevenly distributed data, which were tested statistically with the Mann-Whitney $U$-test, Student's $t$-test or the Wilcoxon rank-sum test, respectively. A p-value $<0.05$ was considered significant. Categorical data are described as frequencies with percentages and were analyzed with the chi-square test or fisher exact test, as appropriate.

The SPSS 20.0 (IBM, Armonk, NY, USA) software was used to perform all of the statistical analyses.

\section{Results}

A total of 689 pediatric patients who had undergone elective Roux-en-Y hepaticojejunostomy during the 11-year study period (from January 2007 to April 2018) met the inclusion criteria and entered into the final analysis (Table 1). Demographic data and postoperative outcomes were available for all patients included in this study. After adjusting for patient weight and the duration of the operation, the average amount of urine output among the entire cohort was $6.01 \mathrm{~mL} / \mathrm{kg}^{*} \mathrm{~h}$, corresponding to a coefficient of variation (COV) of $76.8 \%$. According to the average corrected urine output during the intervention period, patients were dichotomized as "High urine output group $\left(>6.01 \mathrm{~mL} / \mathrm{kg}^{*} \mathrm{~h}\right)$ " or "Low urine output group $\left(<6.01 \mathrm{~mL} / \mathrm{kg}^{*} \mathrm{~h}\right)$ " urine output groups (Table 1) for the purpose of analysis.

There were no significant differences between the two groups regarding basic demographic parameters, comorbidities, American Society of Anesthesiologists (ASA) physical status classification, etc. The number of patients who received an intraoperative blood transfusion was significantly higher in the high urine output group $(\mathrm{p}=0.045)$, although intraoperative blood loss (EBL) was similar in both groups (Table 1). Patients were also comparable in terms of baseline biochemical laboratory parameters and physiologic variables (Table 1). With regard to the CVP, there was no significant difference between the two groups throughout the operation. Additionally, the target MAP was achieved in most cases with no differences between the groups (data not shown). The patients in the higher urine output group had a proportionally greater amount of crystalloids used (12.99 \pm 6.52 and 17.36 $\pm 7.74, p=0.001)$, whereas volumes of colloidal fluids and total fluid balance were not different between the two groups (Table 2).

At any postoperative time-point, the number of participants fulfilling the creatinine criteria for AKIN stage $1 \mathrm{AKI}$ was $56 / 344(16.3 \%)$ versus $59 / 345(17.1 \%)$ in the low versus high groups; for stage 2 , the numbers were $13 / 344$ (3.8\%) versus $11 / 345$ (3.2\%); there were no occurrences of stage $3 \mathrm{AKI}$, all the AKI improved by administering diuretics. Overall, there were no differences in the vasopressor support, diuresis usage and the number of intraoperative hypotensive events, hypokalemic episodes, metabolic acidosis (defined by low bicarbonate) or other laboratory and hemodynamic parameters between the high and low urine output groups (Table 3). There were almost identical kinetics and absolute values in the two groups of any of the investigated inflammatory markers (leukocyte count, fever, 
Table I Baseline Demographics of Eligible Patient and Preoperative Variables

\begin{tabular}{|c|c|c|c|}
\hline & \multicolumn{3}{|l|}{ Total Population } \\
\hline & Low Urine Output Group (344) & High Urine Output Group (345) & $\mathbf{p}$ values \\
\hline Age (yrs) & $4.66 \pm 2.98$ & $4.38 \pm 3.14$ & 0.24 \\
\hline Male:female & $95: 249$ & $99: 246$ & 0.41 \\
\hline Weight (kg) & $15.53 \pm 4.26$ & $14.82 \pm 3.75$ & 0.25 \\
\hline Angiocholitis, n (\%) & $69(20.05)$ & $77(22.32)$ & 0.26 \\
\hline Operative time (mins) & $142.18 \pm 52.63$ & $139.28 \pm 47.75$ & 0.18 \\
\hline $\mathrm{HB}$ on admission & $9.96 \pm 1.32$ & $9.7 I \pm I .4 I$ & 0.28 \\
\hline Estimated blood loss (EBL) & $32.1 \pm 26.86$ & $40.0 \pm 24.85$ & 0.31 \\
\hline Transfused patients, N (\%) & $120(34.88)$ & I43 (4I.45) & 0.045 \\
\hline WBC (I09/L) & $11.36 \pm 5.10$ & $12.14 \pm 4.96$ & 0.15 \\
\hline PCT (ng/mL, normal value: $0-0.5$ ) & $7.12 \pm 2.34$ & $6.75 \pm 2.28$ & 0.13 \\
\hline CPR (mg/L, normal value: $0-10)$ & $18.14 \pm 6.53$ & $20.25 \pm 5.98$ & 0.33 \\
\hline Albumin (g/L, normal range, $35-50$ ) & $32.68 \pm 4.86$ & $33.24 \pm 4.96$ & 0.28 \\
\hline \multicolumn{4}{|l|}{ Operation type, N (\%) } \\
\hline Laparoscopic & $48(13.95)$ & $53(I 5.36)$ & \\
\hline Open & $296(86.05)$ & $292(84.64)$ & 0.34 \\
\hline BUN on admission (mmol/L) & $2.26 \pm 1.46$ & $2.01 \pm 1.10$ & 0.23 \\
\hline Creatinine on admission $(\mu \mathrm{mol} / \mathrm{L})$ & $29.52 \pm 8.55$ & $28.81 \pm 7.52$ & 0.25 \\
\hline Operative blood loss (mL) & $39.14 \pm 16.58$ & $43.14 \pm 15.69$ & 0.28 \\
\hline \multicolumn{4}{|l|}{ ASA classification } \\
\hline ASAI-2 & $201(58.43)$ & $193(56.10)$ & 0.28 \\
\hline ASA3-4 & $143(41.57)$ & $152(43.90)$ & \\
\hline Average CVP (mmHg) & $8.93 \pm 3.51$ & $8.98 \pm 4.16$ & 0.52 \\
\hline Average MAP & & & \\
\hline
\end{tabular}

$\mathrm{C}$ reactive protein [data not shown]) throughout the perioperative period.

A trend for accelerated recovery of gastrointestinal function was noted in patients with low urine output compared

Table 2 Volume of Crystalloid and Fluids Infused

\begin{tabular}{|l|l|l|l|}
\hline $\begin{array}{l}\text { Intraoperative } \\
\text { Fluids }\end{array}$ & $\begin{array}{l}\text { Low Urine } \\
\text { Output } \\
\text { Group (344) }\end{array}$ & $\begin{array}{l}\text { High Urine } \\
\text { Output } \\
\text { Group (345) }\end{array}$ & p values \\
\hline IV crystalloids, $\mathrm{mL} / \mathrm{kg}^{*} \mathrm{~h}$ & $12.99 \pm 6.52$ & $17.36 \pm 7.74$ & 0.001 \\
Colloid, $\mathrm{n}(\%)$ & $246(71.5 \mathrm{I})$ & $257(74.49)$ & 0.21 \\
IV colloids, $\mathrm{mL}$ & $157.34 \pm 85.75$ & $146.49 \pm 97.64$ & 0.19 \\
Urine output, $\mathrm{mL} / \mathrm{kg} \mathrm{g}^{* h}$ & $3.24 \pm 2.76$ & $10.81 \pm 3.22$ & 0.000 \\
Fluid Balance & $218.92 \pm 185.54$ & $227.58 \pm 168.26$ & 0.68 \\
\hline
\end{tabular}

Notes: Fluid Balance $=[$ Crystalloid $+1.5 *($ colloid+blood product $)](\text { Volume In })^{-}$ [Urine Output+3*(estimated blood loss)] (Volume Out)- [Operative duration(hrs)* (weight $(\mathrm{kg})+40)$ ] (Maintenance). with high urine output, as indicated by the first flatus $(p=0.015)$ and first bowel movement $(p=0.008)$. In the low urine output cases, $77.8 \%$ (138/344) of patients spontaneously passed stool within $72 \mathrm{hrs,} \mathrm{whereas} \mathrm{only} 47.1 \%$ (113/ 345) cases in the high urine output passed stool within the same period ( $R R, 1.38 ; 95 \% \mathrm{CI}, 1.01-1.88, \mathrm{p}=0.027)$. The incidences of abdominal distention within 5 PODs in the patients were comparable between the two groups. Patients with a low urine output suffered almost equally from nausea, vomiting, diarrhea and abdominal cramps events as patients with a high urine output in 5 PODs (Table 4).

It is worth noting that the grade II postoperative complications increased in patients with a high urine output compared with those with a low urine output, including anastomotic leakage, infectious complications of pneumonia, incision dehiscence, intraperitoneal abscess, sepsis, and surgical site infections, etc. Forty-seven patients (47/344, 
Table 3 Intraoperative Variables According to the Low and High Urine Output

\begin{tabular}{|l|l|l|l|}
\hline & $\begin{array}{l}\text { Low Urine } \\
\text { Output } \\
\text { Group (344) }\end{array}$ & $\begin{array}{l}\text { High Urine } \\
\text { Output } \\
\text { Group (345) }\end{array}$ & p values \\
\hline $\begin{array}{l}\text { AKI } \\
\text { AKIN stage I } \\
\text { AKIN stage 2 }\end{array}$ & $\begin{array}{l}56(16.28) \\
13(3.78)\end{array}$ & $\begin{array}{l}59(17.10) \\
\text { II (3.19) }\end{array}$ & $\begin{array}{l}0.43 \\
0.42\end{array}$ \\
\hline $\begin{array}{l}\text { Hypotensive } \\
\text { events, n(\%) }\end{array}$ & $36(10.47)$ & $4 \mathrm{I}(1 \mathrm{I} .88)$ & 0.32 \\
\hline $\begin{array}{l}\text { Norepinephrine } \\
\text { usage, n(\%) }\end{array}$ & $48(13.95)$ & $42(12.17)$ & 0.28 \\
\hline Furosemidum, n(\%) & $34(9.88)$ & $39(11.30)$ & 0.32 \\
\hline $\begin{array}{l}\text { Metabolic } \\
\text { acidosis, n(\%) }\end{array}$ & $1 \mathrm{I}(3.20)$ & $17(4.93)$ & 0.039 \\
\hline $\begin{array}{l}\text { Hypokalemic } \\
\text { episodes, n(\%) }\end{array}$ & $3.5 \pm 0.37$ & $3.4 \pm 0.42$ & 0.079 \\
\hline
\end{tabular}

$12.0 \%$ ) in the lower urine output group experienced at least 1 complication compared with $65(65 / 345,19.6 \%)$ in the higher urine output group, with an odds ratio (OD) of 0.68 (95\% confidence interval [95\% CI, 0.45-1.03]; $\mathrm{P}=0.041$ ) (Table 4). The mean length of hospital stay was $8.01 \pm 2.31$ days in patients with a high urine output, which was significantly longer than the mean length of stay $(7.59 \pm 1.24$ days) in patients with a low urine output $(\mathrm{p}=0.016)$. In fact, very similar values were found in nutritional variables and the serum albumin between the groups at all time periods (data not shown).

\section{Discussion}

Current data from a tertiary care hospital showed that low urine output was associated with significant improvements in recovery measures and that it did not increase the risks of compromised kidney health among patients following Roux-en-Y hepaticojejunostomy surgery. Furthermore, the amount of intravenous fluid administered during surgery were significantly decreased in the patients having a low urine output. The total number of complications was also decreased in patients with a low urine output.

Regarding intraoperative fluid management, the basic parameters (blood pressure, heart rate) have been widely monitored during major surgeries. However, treatment should be individualized, tailored to the patients' actual need using appropriate hemodynamic targets rather than simply following the treatment protocol based on MAP or CVP, which may be beneficial for some but may harm others. ${ }^{14-16}$ Intraoperative urine output may be a measure of this goal, in clinical practice, and has been previously investigated as a predictive marker of postoperative AKI. ${ }^{17,18}$ This study expanded previous work by examining the clinical urine output status as an intraoperative fluid monitoring parameter and its association with clinical outcomes across a cohort of pediatric patients undergoing Roux-en-Y hepaticojejunostomy surgery. In the current research, patients with higher urine output received more crystalloid fluid administration. Indeed, intraoperative low diuresis often initiates several actions, and intravenous fluids administration has been the most common solution. ${ }^{19}$ This outcome is similar to a recent paper, in which fluid bolus administration was significantly reduced by changing the minimum urine output target. ${ }^{9}$ The present study demonstrated significant variation in urine output among pediatric patients undergoing gastroenterological surgery. Patient-level variations in fluid administration may be subject to some confounding and selection bias due to patient and operative factors. This fact and our current results

Table 4 Patient Outcomes According to the Low and High Urine Output

\begin{tabular}{|l|l|l|l|l|}
\hline & $\begin{array}{l}\text { Low Urine Output Group } \\
\mathbf{( 3 4 4 )}\end{array}$ & $\begin{array}{l}\text { High Urine Output Group } \\
\mathbf{( 3 4 5 )}\end{array}$ & p values & $\begin{array}{l}\text { Odds Ratio (95\% } \\
\mathbf{C l})\end{array}$ \\
\hline First defecation (days) & $3.01 \pm 1.22$ & $3.19 \pm 1.26$ & 0.18 & \\
First flatus & $3.58 \pm 0.87$ & $4.12 \pm 1.02$ & 0.015 & 0.008 \\
First bowel movement & $2.61 \pm 0.92$ & $3.14 \pm 1.02$ & 0.027 & $1.375(1.007-1.878)$ \\
Stool within 72 hrs & $138(40.12)$ & $113(32.75)$ & 0.31 & \\
Abdominal distention & $51(14.83)$ & $59(17.10)$ & 0.075 & $1.369(0.917-2.043)$ \\
Nausea or vomiting events & $66(19.19)$ & $51(14.78)$ & 0.14 & \\
Diarrhea and abdominal cramps & $56(16.28)$ & $68(19.71)$ & 0.041 & $0.682(0.453-1.026)$ \\
No.of patients with complications, $\mathrm{n}(\%)$ & $47(13.66)$ & $65(18.84)$ & 0.016 & \\
Length of stay (d) & $7.59 \pm 1.24$ & $8.01 \pm 2.31$ & \\
\hline
\end{tabular}


support the notion that traditional and standard fluid strategies are not appropriate at the individual level and reiterate the need for individual fluid support to appropriately adjust urine output. Real opportunities still exist to standardize behavior at the hospital level if better practices can be identified and implemented.

The results of our study indicate a lack of correlation between intraoperative urine output and postoperative AKI. We could not identify any early adverse effects from the use of crystalloid solution as indicated by renal function tests. In recent randomized studies, no correlation was found between intraoperative fluid management and AKI. ${ }^{20-22}$ Additionally, in another research, kidney function, as measured by serum cystatin $\mathrm{C}$, serum creatinine, and direct measures of renal perfusion and glomerular filtration was also unaffected by lowering the urine output target, which is in line with present findings. ${ }^{9}$ Restrictive fluid management was not predictive of AKI nor was target oliguria reversal able to prevent AKI. ${ }^{23-25}$ In previous restrictive versus liberal intraoperative maintenance fluid therapy trials, no association between observed intraoperative urine output and postoperative renal dysfunction was found. ${ }^{19,26}$ It has previously been believed that restrictive resuscitation is associated with increased AKI. However, analysis of urine in this study did not confirm these beliefs. ${ }^{27}$

As liberal fluid administration may correlate with a worsened postoperative outcome as suggested by several studies, the recommendation to infuse fluid to maintain, among other things, a urine output of at least $0.5 \mathrm{~mL} / \mathrm{kg} / \mathrm{h}$ has been reconsidered. ${ }^{20,21}$ Even in recent research, the perioperative urine output target of $0.2 \mathrm{~mL} / \mathrm{kg} / \mathrm{h}$ is noninferior to the standard target of $0.5 \mathrm{~mL} / \mathrm{kg} / \mathrm{h}$ and results in a large intravenous fluid sparing. ${ }^{9}$ In other words, urine output might be a "softer" marker of acute kidney injury than changes in serum biochemistry. It is therefore logical to conclude that the current urine output practice in anesthetized pediatric patients may be overstated in terms of kidney injury. As mentioned earlier, hypotension and hypovolemia are important causes of AKI. The evaluation of decreased urine output and AKI should be guided by the patient's history and presentation of the condition. It is important to approach acute kidney injury in a stepwise fashion, considering the most common and most easily remedied causes first. Several studies have investigated the mechanism for the decreased intraoperative urine output and found that the reduced clearance and a slower distribution of infused $0.9 \%$ saline during general anesthesia was only a small fraction of that observed in conscious patients and volunteers. Anesthesia-induced vasodilatation, blood pressure and body temperature fluctuations, activation of the renin-angiotensin-aldosterone axis, antidiuretic hormone and psychological stress may contribute to decreased intraoperative urine output. ${ }^{28-30}$

There is a difference in metabolic acidosis rate with increased incidence of acidosis in the higher urine output group. Based on the current data, I completely agree with your comment about this. The high crystalloid administration might result in hyperchloremia, which should account for the increased incidence of acidosis. Although choledochal cyst resection is associated with an uneventful recovery in most patients, the postoperative gastroenterological recovery and complications might be different in terms of perioperative care. Postoperative intestinal recovery is the main focus of gastroenterological surgery, which is the most important reason of the postoperative length of stay. ${ }^{31}$ In clinical practice, a considerable quantity of fluid is administered to sustain the current urine output. Here, we also evaluated for the first time if urine output is associated with postoperative intestinal function recovery. In this research, we detected an increased total number of complications and unfavorable postoperative outcomes following an increase in urine output, which was closely related to intraoperative crystalloids administration. We performed this measurement during the monitoring setting. The clinically intestinal complaints that correlated with intestinal function should be adequately monitored as much as possible. In this research, it is indicative of the remarkable beneficial effects of low urine output for postoperative gastrointestinal recovery of defecation and oral solid diet. These results might be due to excessive fluid therapy. Taken together with previous studies, ${ }^{9,19}$ the present data highlighted the benefits of low urine output, which might be associated with a restrictive fluid therapy in pediatric patients undergoing major surgery. Because this strategy is still not widely used in current pediatric surgical practices, evidence-based best practices should be implemented to promote a safer, higher quality of patient care. In fact, in our institute, we have implemented the fluid therapy strategy using more tangible hemodynamic parameters of urine output.

There are some limitations to our study. First, the data were collected locally from a single institution, and although we did perform a power analysis, this was still a relatively small single center study. Our study has limitations inherent to the retrospective database analysis where unmeasured differences, known selection and 
treatment bias may contribute to confounding, which may limit the generalizability of the results. The general surgery procedures were performed in our hospital over a long period of time; therefore, there may have been many practice changes within both surgical and the ICU divisions, leading to different care practices between study patients, which may not reflect the outcomes from current treatment algorithms. The urine output variability in this research might be potentially impacted by some residual confounders we have not mentioned here. For example, some hemodynamic parameters were not evaluated, which could have been important in determining the amount and type of fluids administered. On the other hand, the main indicators for AKI are general clinical biochemical measurements. These may be improved by including more biomarkers of kidney damage, such as neutrophil gelatinase-associated lipocalin (NGAL), serum cystatin $\mathrm{C}$ and a larger sample of patients to detect subtle degrees of kidney damage. There were increased blood transfusions in the high urine output group. That is to say, the negative impact of blood transfusion beyond simply volume administration may impact post-surgical outcomes. The relation between urine output and blood transfusion should be further investigated in new research project.

\section{Conclusions}

In conclusion, a wide range and significant urine output variability were noted for pediatric patients undergoing abdominal surgery. In the current study, high urine output might be associated with more intraoperative fluid administration and low urine output in the current practice provided some beneficial postoperative recovery, without resulting in an increase in renal injury in pediatric patients undergoing hepaticojejunostomy, although this conclusion should be evaluated further. Our future studies will focus on the optimal amount of urine output target to improve clinical quality of care, which should be implemented in the best practice guidelines of fluid administration.

\section{Ethics Approval and Consent to Participate}

This study was approved and supervised by the ethics committee of the Children's Hospital of Chongqing Medical University and was performed in accordance with the ethical standards of the 1964 Declaration of Helsinki. Informed written consent was obtained from all patients.

\section{Consent for Publication}

All the patient data used in this study were approved by the guardian, and the informed consent forms were signed.

\section{Data Sharing Statement}

The datasets analyzed in the study are available from the corresponding author on reasonable request.

\section{Acknowledgments}

We thank Prof. Xianqing Jin for providing technical assistance and for the insightful discussions during the preparation of the manuscript. We thank Dr Xiaoyong Zhang at the Wistar Institute, USA, for helping with the linguistic revision of the manuscript.

\section{Author Contributions}

All authors contributed to data analysis, drafting or revising the article, gave final approval of the version to be published, and agree to be accountable for all aspects of the work.

\section{Funding}

The research was supported by National Natural Science Foundation of China (No: 30973440, 30770950), and the Key Project of the Chongqing Natural Science Foundation (CSTC, 2008BA0021, cstc2012jjA0155).

\section{Disclosure}

The authors declare that they have no competing interests.

\section{References}

1. Benes J, Chytra I, Altmann P, et al. Intraoperative fluid optimization using stroke volume variation in high risk surgical patients: results of prospective randomized study. Crit Care. 2010;14(3):R118. doi:10.11 $86 / \operatorname{cc} 9070$

2. Holte K, Klarskov B, Christensen DS, et al. Liberal versus restrictive fluid administration to improve recovery after laparoscopic cholecystectomy: a randomized, double-blind study. Ann Surg. 2004;240 (5):892-899. doi:10.1097/01.sla.0000143269.96649.3b

3. Lilot M, Ehrenfeld JM, Lee C, Harrington B, Cannesson M, Rinehart J. Variability in practice and factors predictive of total crystalloid administration during abdominal surgery: retrospective two-centre analysis. Br J Anaesth. 2015;114(5):767-776. doi:10.10 93/bja/aeu452

4. Goren O, Levy A, Cattan A, Lahat G, Matot I. Acute kidney injury in pancreatic surgery; association with urine output and intraoperative fluid administration. Am J Surg. 2017;214(2):246-250. doi:10.1016/j. amjsurg.2017.01.040

5. Voldby AW, Brandstrup B. Fluid therapy in the perioperative setting a clinical review. J Intensive Care. 2016;16(4):27. doi:10.1186/ s40560-016-0154-3

6. Cannesson M, Pestel G, Ricks C, Hoeft A, Perel A. Hemodynamic monitoring and management in patients undergoing high risk surgery: a survey among North American and European anesthesiologists. Crit Care. 2011;15(4):R197. doi:10.1186/cc10364 
7. Makaryus R, Miller TE, Gan TJ. Current concepts of fluid management in enhanced recovery pathways. Br J Anaesth. 2018;120 (2):376-383. doi:10.1016/j.bja.2017.10.011

8. Chappell D, Jacob M, Hofmann-Kiefer K, Conzen P, Rehm M. A rational approach to perioperative fluid management. Anesthesiology. 2008;109 (4):723-740. doi:10.1097/ALN.0b013e3181863117

9. Puckett JR, Pickering JW, Palmer SC, et al. Low versus standard urine output targets in patients undergoing major abdominal surgery: a randomized noninferiority trial. Ann Surg. 2017;265(5):874-881. doi:10.1097/SLA.0000000000002044

10. Sinnatamby C, Edwards CR, Kitau IMH. Antidiuretic hormone response to high and conservative fluid regimes in patients undergoing operation. Surg Gynecol Obstet. 1974;139(5):715-719.

11. Albåge A, van der Linden J, Bengtsson L, Lindblom D, Kennebäck G, Berglund $\mathrm{H}$. Elevations in antidiuretic hormone and aldosterone as possible causes of fluid retention in the Maze procedure. Ann Thorac Surg. 2001;72(1):58-64. doi:10.1016/S0003-4975(01)02688-1

12. Regenbogen SE, Shah NJ, Collins SD, Hendren S, Englesbe MJ, Campbell DA Jr. Population-based assessment of intraoperative fluid administration practices across three surgical specialties. Ann Surg. 2017;265(5):930-940. doi:10.1097/SLA.0000000000001745

13. Kellum JA, Lameire N; KDIGO AKI Guideline Work Group. Diagnosis, evaluation, and management of acute kidney injury: a KDIGO summary (Part 1). Crit Care. 2013;17:204. doi:10.1186/cc11454

14. Kirov MY, Kuzkov VV, Molnar Z. Perioperative haemodynamic therapy. Curr Opin Crit Care. 2010;16:384-392. doi:10.1097/ MCC.0b013e32833ab81e

15. Drage S, Boyd O. Perioperative goal directed haemodynamic therapy - do it, bin it, or finally investigate it properly? Crit Care. 2007;11(5):170. doi:10.1186/cc6130

16. Benes J, Zatloukal J, Simanova A, Chytra I, Kasal E. Cost analysis of the stroke volume variation guided perioperative hemodynamic optimization - an economic evaluation of the SVVOPT trial results. BMC Anesthesiol. 2014;22(14):40. doi:10.1186/1471-2253-14-40

17. Parolari A, Pesce LL, Pacini D, et al., Monzino Research Group on Cardiac Surgery Outcomes. Risk factors for perioperative acute kidney injury after adult cardiac surgery: role of perioperative management. Ann Thorac Surg. 2012;93(2):584-591. doi:10.1016/j.athoracsur.2011.09.073

18. Song Y, Kim DW, Kwak YL, et al. Urine output during cardiopulmonary bypass predicts acute kidney injury after cardiac surgery: a single-center retrospective analysis. Medicine (Baltimore). 2016;95 (22):e3757. doi:10.1097/MD.0000000000003757

19. Matot I, Dery E, Bulgov Y, Cohen B, Paz J, Nesher N. Fluid management during video-assisted thoracoscopic surgery for lung resection: a randomized, controlled trial of effects on urinary output and postoperative renal function. J Thorac Cardiovasc Surg. 2013;146 (2):461-466. doi:10.1016/j.jtcvs.2013.02.015

20. Alpert RA, Roizen MF, Hamilton WK, et al. Intraoperative urinary output does not predict postoperative renal function in patients undergoing abdominal aortic revascularization. Surgery. 1984;95(6):70 $7-711$.

Therapeutics and Clinical Risk Management

\section{Publish your work in this journal}

Therapeutics and Clinical Risk Management is an international, peerreviewed journal of clinical therapeutics and risk management, focusing on concise rapid reporting of clinical studies in all therapeutic areas, outcomes, safety, and programs for the effective, safe, and sustained use of medicines. This journal is indexed on PubMed Central, CAS,
21. Kheterpal S, Tremper KK, Englesbe MJ, et al. Predictors of postoperative acute renal failure after noncardiac surgery in patients with previously normal renal function. Anesthesiology. 2007;107 (6):892-902. doi:10.1097/01.anes.0000290588.29668.38

22. Matot I, Paskaleva R, Eid L, et al. Effect of the volume of fluids administered on intraoperative oliguria in laparoscopic bariatric surgery: a randomized controlled trial. Arch Surg. 2012;147(3):228-234. doi:10.1001/archsurg.2011.308

23. Thirlby RC. Is my patient wet or dry? Should my patient be wet or dry? A first step in answering these queries: comment on "Effect of the volume of fluids administered on intraoperative oliguria in laparoscopic bariatric surgery". Arch Surg. 2012;147(3):234-235. doi:10. 1001/archsurg.2011.1413

24. Egal M, Erler NS, de Geus HR, van Bommel J, Groeneveld AB. Targeting oliguria reversal in goal-directed hemodynamic management does not reduce renal dysfunction in perioperative and critically ill patients: a systematic review and meta-analysis. Anesth Analg. 2016;122(1):173-185. doi:10.1213/ANE.00000000 00001027

25. Leedahl DD, Frazee EN, Schramm GE, et al. Derivation of urine output thresholds that identify a very high risk of AKI in patients with septic shock. Clin J Am Soc Nephrol. 2014;9(7):1168-1174. doi:10.2215/CJN.09360913

26. Wenkui Y, Ning L, Jianfeng G, et al. Restricted peri-operative fluid administration adjusted by serum lactate level improved outcome after major elective surgery for gastrointestinal malignancy. Surgery. 2010;147(4):542-552. doi:10.1016/j.surg.20 09.10 .036

27. Mason SA, Nathens AB, Finnerty CC, et al., Inflammation and the Host Response to Injury Collaborative Research Program. Hold the pendulum: rates of acute kidney injury are increased in patients who receive resuscitation volumes less than predicted by the Parkland equation. Ann Surg. 2016;264(6):1142-1147. doi:10.1097/SLA.000 0000000001615

28. Norberg A, Hahn RG, Li H, et al. Population volume kinetics predicts retention of $0.9 \%$ saline infused in awake and isoflurane-anesthetized volunteers. Anesthesiology. 2007;107 (1):24-32. doi:10.1097/01.anes.0000268387.34758.6d

29. Hahn RG. Volume kinetics for infusion fluids. Anesthesiology. 2010;113:470e481. doi:10.1097/ALN.0b013e3181dcd88f

30. Olsson J, Svensen CH, Hahn RG. The volume kinetics of acetated ringer's solution during laparoscopic cholecystectomy. Anesth Analg. 2004;99:1854e1860.

31. Sun Y, Chai F, Pan C, Romeiser JL, Gan TJ. Effect of perioperative goal-directed hemodynamic therapy on postoperative recovery following major abdominal surgery - a systematic review and metaanalysis of randomized controlled trials. Crit Care. 2017;21:141. doi:10.1186/s13054-017-1728-8 a very quick and fair peer-review system, which is all easy to use. Visit http://www.dovepress.com/testimonials.php to read real quotes from published authors.

\section{Dovepress}

